\title{
Do Alternative Cooling Methods Have Effective Cooling Rates for Hyperthermia Compared With Previously Established CWI Cooling Rates?
}

\author{
Kailin C. Parker, Rachel R. Shelton, and Rebecca M. Lopez
}

\begin{abstract}
Clinical Scenario: In the last few years, there have been several studies examining alternative cooling strategies in the treatment of exertional heat stroke (EHS). Morbidity and mortality with EHS are associated with how long the patient's core body temperature remains above the critical threshold of $40.5^{\circ} \mathrm{C}$. Although cold-water immersion (CWI) is the gold standard of treatment when cooling a patient with EHS, more recent alternative cooling techniques have been examined for use in settings where CWI may not be feasible (ie, remote locations). Clinical Question: Do alternative cooling methods have effective core body temperature cooling rates for hyperthermia compared with previously established CWI cooling rates? Summary of Key Findings: The authors searched for studies using alternative cooling methods to cool hyperthermic individuals. To be included, the studies needed a PEDro score $\geq 6$ and a level of evidence $\geq 2$. They found 9 studies related to our focused clinical question; of these, 5 studies met the inclusion criteria. The cooling rates for hand cooling, cold-water shower, and ice-sheet cooling were $0.03^{\circ} \mathrm{C} / \mathrm{min}, 0.08^{\circ} \mathrm{C} / \mathrm{min}$, and $0.06^{\circ} \mathrm{C} / \mathrm{min}$, respectively, whereas the tarp-assisted cooling with oscillation (TACO) method was the only method that had an acceptable cooling rate (range $0.14-0.17^{\circ} \mathrm{C} / \mathrm{min}$ ). Clinical Bottom Line: When treating EHS, if CWI is not available, the tarp-assisted cooling method may be a reasonable alternative. Clinicians should not use cold shower, hand cooling, or ice-sheet cooling if better cooling methods are available. Clinicians should always use CWI when available. Strength of Recommendation: Five level 2 studies with PEDro scores $\geq 6$ suggest the TACO method is the only alternative cooling method that decreases core body temperature at a similar, though slower, rate of CWI. Hand cooling, cold showering, and ice-sheet cooling do not decrease core body temperature at an appropriate rate and should not be used in EHS situations if a modality with a better cooling rate is available.
\end{abstract}

Keywords: exertional heat stroke, exercise induced hyperthermia, heat illness, emergency management

\section{Clinical Scenario}

Death from exertional heat stroke (EHS) is preventable if the condition is promptly recognized and the patient is aggressively cooled. ${ }^{1,2}$ Morbidity and mortality with EHS are often associated with how long a patient with EHS temperature remains above the critical threshold of $40.5^{\circ} \mathrm{C}\left(105^{\circ} \mathrm{F}\right) .^{1,2}$ Therefore, health care providers who are likely to deal with EHS outside of a hospital setting (ie, athletic, industrial, or military setting) should have a heat illness-specific emergency action plan for the proper recognition of EHS and an effective cooling strategy based on their setting and the available resources. With a cooling rate of about $0.2^{\circ} \mathrm{C} / \mathrm{min}$, cold-water immersion (CWI) is the gold-standard treatment when cooling a patient with EHS. ${ }^{1,2}$ The effectiveness and cooling rate for CWI has been well established in the literature, and several other studies and reviews have compared the cooling rates of various modalities in the past. ${ }^{3-6}$ More recently, however, alternative cooling techniques have been tested due to some settings not having access to a whirlpool or cold tub available for CWI. The tarp-assisted cooling with oscillation (TACO) method, ${ }^{7,8}$ ice-sheet cooling, ${ }^{9}$ cold-water showering, ${ }^{10}$ and hand cooling ${ }^{11}$ are a few of the alternative cooling methods that have been examined as alternative cooling strategies for EHS due to their portability and cost.

The authors are with the Department of Orthopaedics and Sports Medicine, Morsani College of Medicine, University of South Florida, Tampa, FL. Lopez (rlopez@ health.usf.edu) is corresponding author.
Although alternative cooling methods have been proposed, it is unknown if they are an effective alternative to CWI. To date, the newer studies examining these alternative cooling methods had not been critically appraised in the literature. Clinicians who may be responsible for the prehospital care of a patient with EHS are often limited by resources or need to determine alternative cooling strategies due to working in remote locations where CWI is not possible (ie, military or athletic fields not near a school). It is imperative for these clinicians to have the most up-to-date evidence from which to base their clinician decisions for the prehospital care of a patient with EHS.

\section{Focused Clinical Question}

Do alternative cooling methods have effective core body temperature cooling rates for hyperthermia compared with previously established CWI cooling rates?

\section{Summary of Key Findings}

We searched for randomized clinical trials that used alternative methods of cooling that were applied on exercising hyperthermic individuals. Studies examining the efficacy of various cooling modalities cannot be applied on actual patients with EHS; therefore, these studies often examine the cooling rates for hyperthermic individuals rather than patients with EHS. For studies to be included, they had to have a PEDro score of 6 or greater and a 
level of evidence score of 2 or greater based on the Oxford Centre for Evidence-Based Medicine. PEDro scores were evaluated by all 3 authors individually and compared. If differing scores occurred, they were reevaluated until there was agreement between the authors. Allocation being concealed and the blinding of subjects, therapists, and assessors were the only 4 criteria not met when assessing the PEDro scores. We also excluded studies published prior to 2015 to ensure we were only examining recent, highquality studies that had not yet been critically appraised. We found 7 studies that were related to our focused clinical question and examined the efficacy of alternative cooling rates. Of the 7 that were found, 5 studies met the inclusion and exclusion criteria. ${ }^{7-11}$ When comparing these 5 studies with CWI, TACO was the only alternative cooling method that had a similar cooling rate. Cooling rates of hand cooling, cold-water shower, and ice-sheet cooling were $0.03^{\circ} \mathrm{C} / \mathrm{min},{ }^{11} 0.078^{\circ} \mathrm{C} / \mathrm{min},{ }^{10}$ and $0.06^{\circ} \mathrm{C} / \mathrm{min},{ }^{9}$ respectively. The cooling rate in the 2 studies that examined the TACO method ranged between 0.14 and $0.17^{\circ} \mathrm{C} / \mathrm{min},{ }^{7,8}$ which is still a lower cooling rate compared with CWI.

\section{Clinical Bottom Lines}

When treating EHS, the gold standard for cooling is CWI. ${ }^{1,2}$ However, if CWI is not available, the TACO method is a reasonable alternative. The study findings suggest that clinicians should not use a cold shower, hand cooling, or ice-sheet cooling if better cooling methods are available because the published studies have shown that the cooling rates with these modalities are poor. The latest consensus statement on prehospital care of EHS states that the minimum rate of cooling that is necessary for treating a patient with EHS is $0.15^{\circ} \mathrm{C} / \mathrm{min} .{ }^{1}$ Therefore, clinicians should always use CWI (cooling rate of $0.2^{\circ} \mathrm{C} / \mathrm{min}$ ) when available.

\section{Strength of Recommendation}

Five level 2 studies with PEDro scores $\geq 6$ suggest that the TACO method is the only alternative cooling method that decreases core body temperature at a similar rate to CWI. Hand cooling, cold showering, and ice-sheet cooling do not decrease core body temperature at an appropriate rate and should not be used in EHS situations if a modality with a better cooling rate is available.

\section{Search Strategy}

\section{Databases and Sites Searched}

- PubMed

- SPORTDiscus

- Google Scholar

- Review of reference lists

\section{Search Terms}

- Patient/client group: hyperthermia, hyperthermic patients, hyperthermic adults, hyperthermic athletes

- Intervention/Assessment: cooling methods, cooling interventions, alternative cooling, tarp-assisted cooling, arm immersion, water dousing, cold shower, core body temperature

- Outcome: heat illness, heat stroke, exertional heat illness, heat strain, exertional heat stroke

PICO

- P: hyperthermic individuals

- I: alternative cooling methods

- C: cold-water immersion

- O: cooling rate

\section{Search Limits: Predetermined Inclusion and Exclusion Criteria}

\section{Inclusion}

- Alternative cooling methods for hyperthermic individuals

- Studies that used a valid core body temperature measure: rectal temperature, esophageal temperature, and/or gastrointestinal ingestible thermistor

- Limited studies to those published between 2015 and 2018

- Only included studies classified as level 2 evidence on the Oxford Centre for Evidence-Based Medicine

- PEDro scores of $\geq 6$

\section{Exclusion}

- Studies using passive heating

- Studies that did not use water or ice as a cooling method

- Studies that were not randomized controlled trials

- Studies not using human subjects

\section{Results of Search}

We searched for randomized control trials of hyperthermic individuals who exercised in the heat (either controlled or outdoors) and were cooled with an alternative cooling method. The original search yielded 143 results. After reviewing abstracts, 134 were eliminated. Of the remaining 9 papers, 5 studies $^{7-11}$ met our criteria and are presented in Tables 1 and 2.

- Clinical trials were researched and chosen based on a PEDro score of $6 / 10$ or higher or level 2 evidence that studied hyperthermia alternative cooling methods.

Table 1 Study Design Summary

\begin{tabular}{|c|c|c|c|}
\hline Reference & Study design & PEDro score & Level of evidence \\
\hline Hosokawa et $\mathrm{al}^{7}$ & Randomized controlled trial & 6 & 2 \\
\hline Luhring et $\mathrm{al}^{8}$ & Randomized controlled trial & 6 & 2 \\
\hline Butts et $\mathrm{al}^{9}$ & Randomized controlled trial & 6 & 2 \\
\hline Butts et $\mathrm{al}^{10}$ & Randomized controlled trial & 6 & 2 \\
\hline Adams et $\mathrm{al}^{11}$ & Randomized controlled trial & 6 & 2 \\
\hline
\end{tabular}




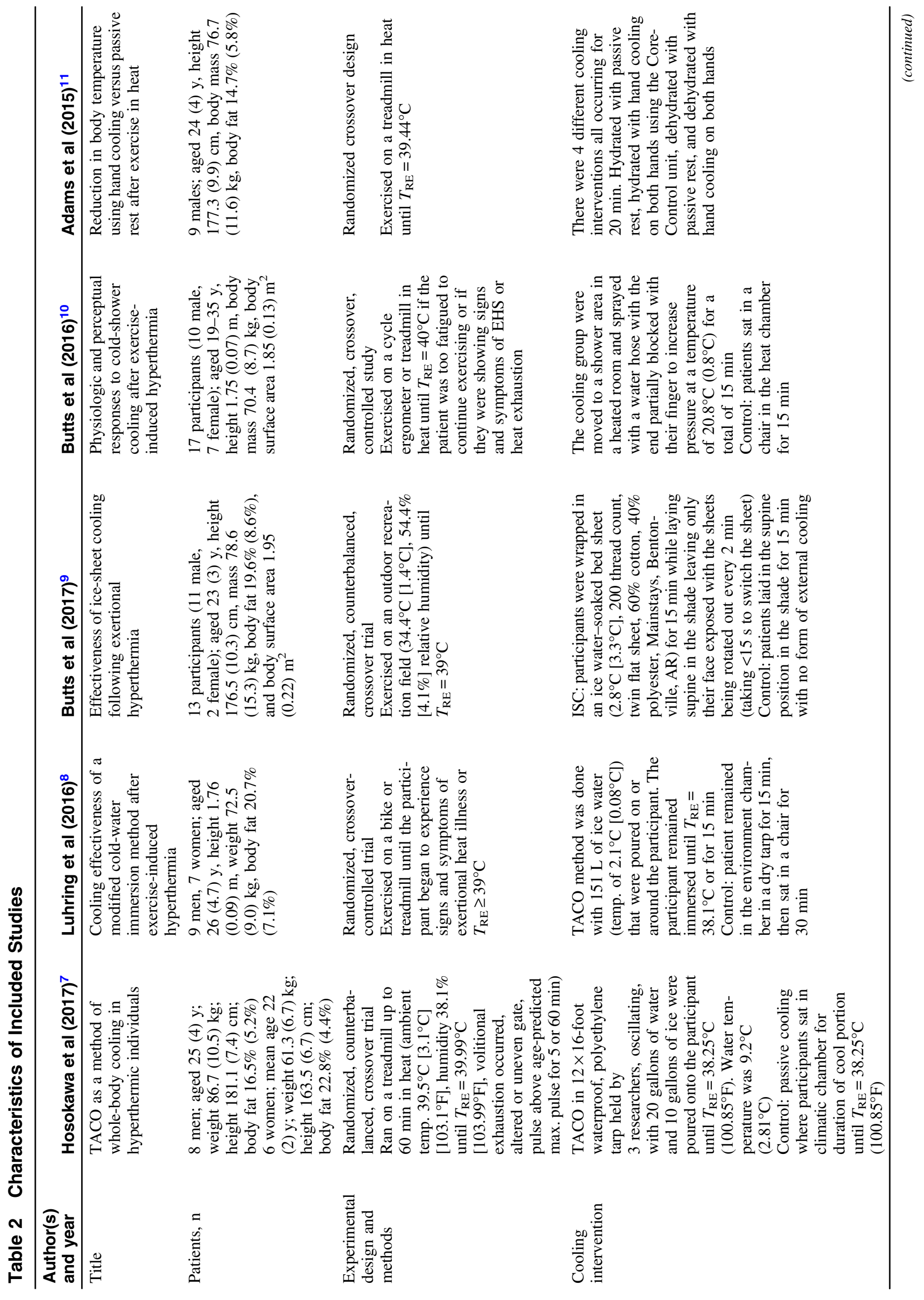




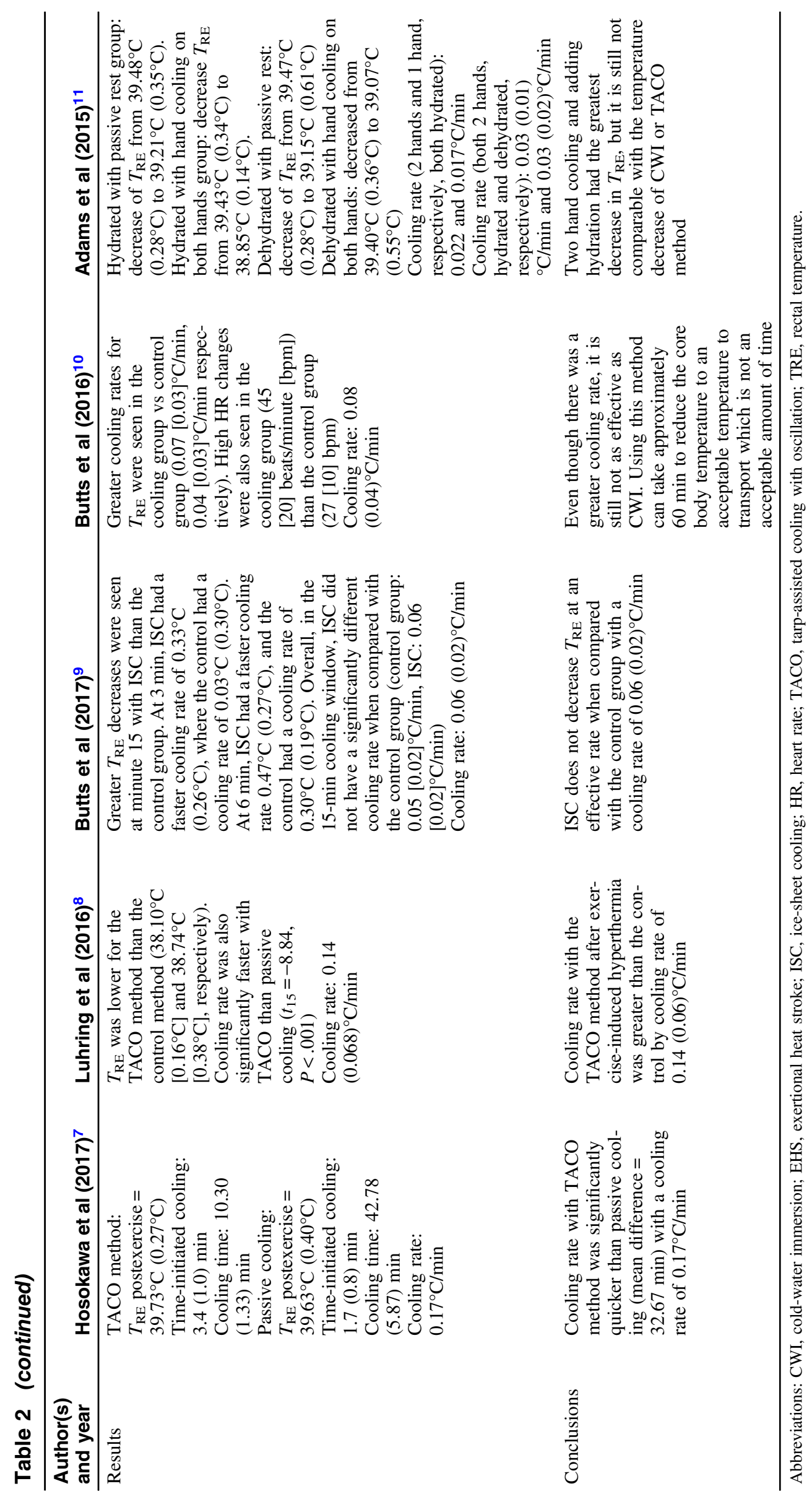


- Seven studies were included in the initial search; however, only 5 studies met the inclusion and exclusion criteria.

- All 5 studies were randomized controlled trials examining the effectiveness of a cooling modality on hyperthermic human subjects.

- Two of the 5 examined the effectiveness of TACO. ${ }^{7,8}$

- One of the 5 studies looked at the effectiveness of ice-sheet cooling, ${ }^{9}$ one study looked at the bodies' response to coldshower cooling, ${ }^{10}$ and the final study looked at the decreased body temperature on hyperthermic individuals using hand cooling. ${ }^{11}$

- Two studies found a similar cooling rates to CWI in decreasing core body temperature. ${ }^{7,8}$

- Three studies did not have significant cooling rates (compared with CWI) when cooling hyperthermic individuals. ${ }^{9-11}$

\section{Summary of Best Evidence}

The most recent evidence-based recommendations for the care of EHS state that CWI is the most effective cooling method for patients with EHS. ${ }^{1,2}$ However, if CWI is not an available option due to exercising in a remote location or field or not being able to transport the patient with EHS to the cold tub, the TACO method is a suitable alternative with a mean cooling rate of $0.16^{\circ} \mathrm{C} / \mathrm{min} .^{7,8}$ The cooling rates of ice-sheet cooling $\left(0.06^{\circ} \mathrm{C} / \mathrm{min}\right),{ }^{9}$ single- and double-hand cooling $\left(0.03^{\circ} \mathrm{C} / \mathrm{min}\right),{ }^{11}$ and cold showering $\left(0.078^{\circ} \mathrm{C} / \mathrm{min}\right)^{10}$ are not ideal and may not successfully cool a patient with EHS.

\section{Implication for Practice, Education, and Future Research}

Appraising the studies that examined the use of hand cooling, icesheet cooling, and cold showering revealed that these are not as effective at reducing core body temperature in hyperthermic athletes as CWI and the TACO method. All but one ${ }^{7}$ of the included studies had a cooling rate below the recommended minimum rate of cooling for treating EHS $\left(0.15^{\circ} \mathrm{C} / \mathrm{min}\right) .{ }^{1}$ Rapidly reducing core body temperature in the case of EHS within 30 minutes of collapse is the key to survival. Cooling the temperature of a patient with EHS to below the critical threshold of $40.5^{\circ} \mathrm{C}$ can decrease the risk of morbidity and mortality rates. ${ }^{1}$

The cooling rates for the 2 TACO studies were just above and below this criterion of $0.15^{\circ} \mathrm{C} / \mathrm{min}$, with one study's cooling rate at $0.14^{\circ} \mathrm{C} / \mathrm{min}^{8}$ and the other at $0.17^{\circ} \mathrm{C} / \mathrm{min} .{ }^{7}$ With a mean cooling rate of $0.16^{\circ} \mathrm{C} / \mathrm{min}$, the TACO method of cooling may be an effective alternative for cooling a patient with EHS if CWI was not available. These 2 studies could have had different cooling rates due to differences in study design or methodology (ie, different water temperatures, differences in postexercise core body temperature, or different environmental conditions). For example, in the Hosokawa et $\mathrm{al}^{7}$ study, the maximum core body temperature from the exercise protocol was $39.73^{\circ} \mathrm{C}$, whereas in the Luhring et al, ${ }^{8}$ maximum core body temperature prior to cooling was $39.27^{\circ} \mathrm{C}$. This greater temperature gradient could have resulted in a faster cooling rate in the Hosokawa study. Furthermore, the 2 studies had different criteria for the termination of cooling, with the Luhring et al study stopped cooling once subjects reached $38.1^{\circ} \mathrm{C}$, whereas Hosokawa et $\mathrm{al}^{7}$ stopped cooling at $38.25^{\circ} \mathrm{C}$. In an actual EHS scenario where the patient's temperature is dangerously elevated about $40.5^{\circ} \mathrm{C}$, the cooling rate using the TACO method would be acceptable. In some clinical situations, CWI may not be an option due to space, finances, or other logistics such as a remote field and travel. The TACO method is a simple and economical option for an athletic trainer or other health care provider to use. This cooling method only requires a tarp that is appropriately sized for the patient, ice, water, and 3 or more people to hold the patient in the tarp. This method uses approximately four 10-gallon coolers of ice and water; most athletic settings have this amount available during outdoor activities.

Passive hand cooling with hydration did not decrease core body temperature as efficiently as CWI and should not be used as an alternative cooling method. The rate at which passive doublehand cooling with hydration cooled was $0.03^{\circ} \mathrm{C} / \mathrm{min} .{ }^{11}$ In a real case of EHS, this rate would not decrease the core body temperature within 30 minutes at $38.6^{\circ} \mathrm{C}$ to allow for an increased survival rate. ${ }^{11} \mathrm{With}$ a cooling rate of $0.05^{\circ} \mathrm{C} / \mathrm{min}$, ice-sheet cooling also did not cool as effectively. Such a poor cooling rate used with an actual patient with EHS may increase the chance of organ failure and potentially death. ${ }^{9}$ Cold-shower cooling resulted in surprisingly low cooling rate of $0.078^{\circ} \mathrm{C} / \mathrm{min}^{10}$; this cooling rate may also not be effective in decreasing the risk morbidity or mortality associated with EHS when the patient's hyperthermia is not reduced within 30 minutes. The water used in the shower study was turned to the coldest setting which was $28.28^{\circ} \mathrm{C}\left(4.48^{\circ} \mathrm{C}\right)$ and a hose temperature of $23.4^{\circ} \mathrm{C}\left(1.1^{\circ} \mathrm{C}\right) .{ }^{10}$ If the water in the shower were cooler (approximately $15^{\circ} \mathrm{C}$ ), ${ }^{2}$ the cooling effect could have been improved. This modality could potentially be an effective cooling alternative in settings, where the water in the locker room is colder; therefore, clinicians should determine whether the water temperature at their facility would be cold enough to cool a patient with EHS. It would also be imperative to determine if the water temperature in the shower varies during different times of day (preferably the hottest part of the day in your location). This will help determine whether cold-water showering is a good alternative cooling method for any given setting. ${ }^{6}$

Rapid recognition of signs and symptoms of EHS, as well as rapid cooling, can help save the lives of patients with EHS. ${ }^{1}$ It is imperative for health care providers to be able to recognize the key signs and symptoms of EHS (increased core body temperature $>40.5^{\circ} \mathrm{C}$ and central nervous system dysfunction). Proper on-site treatment via aggressive cooling prior to transport to an emergency department is the key to survival with EHS. ${ }^{1,2,12,13}$ The athletic trainer or other clinician should "cool first, transport second"2 and continuously monitor core body temperature until it is appropriate to safely transport the patient. ${ }^{1}$ The patient with EHS should be cooled until core body temperature reaches the range of $38.6^{\circ} \mathrm{C}$ to $38.9^{\circ} \mathrm{C} .{ }^{1,2}$

Best practice and the key to surviving EHS are to cool a hyperthermic patient in less than 30 minutes from the initial collapse. ${ }^{1}$ A case series of 6 fatal heat strokes in the Israeli military demonstrated the importance of proper triage and treatment for EHS. ${ }^{14}$ In 6 out of the 6 EHS fatalities, there was an absence of medical triage. ${ }^{14}$ Improper diagnosis and inadequate treatment were also cited as common factors leading to death from EHS. ${ }^{14}$ Conversely, there are other examples of EHS cases with a positive outcome. For example, several races have reported multiple cases of EHS over the years with a $100 \%$ survival rate. ${ }^{15,16}$ The medical tent in the Marine Corps Marathon uses water ice therapy, which involves the patient laying on a stretcher that is rested on a tub that is filled with $10^{\circ} \mathrm{C}$ to $12.8^{\circ} \mathrm{C}$ water and 6 to 8 medical persons dousing the entire patient in water. ${ }^{16}$ The patient's head is wrapped with an ice towel, and all major muscle groups are massaged with ice bags until core body temperature reaches $38.9^{\circ} \mathrm{C} .{ }^{16}$ Although the 
cooling rate reported was lower than $\mathrm{CWI}\left(0.13^{\circ} \mathrm{C}\left[0.04^{\circ} \mathrm{C}\right]\right)$, they reported a $100 \%$ survival rate. ${ }^{16}$ The Falmouth Road Race medical tent also used CWI to treat EHS, and they reported 274 EHS cases over the course of 18 years with $100 \%$ survival. ${ }^{15}$ These 2 examples demonstrate how death from EHS can be avoided with proper recognition and treatment on-site. Even if CWI was not an option for the case series of 6 fatal EHSs in a military setting, an alternative cooling method that cools at $0.15^{\circ} \mathrm{C} / \mathrm{min}$ (ie, TACO method, water ice therapy) could have increased the chance of survival.

Any location where exercising individuals could be at risk of EHS should have a heat safety policy in place that includes a cooling protocol. The protocol can include having a cold tub on-site, having a way to transport a patient to a cold tub, or having an alternative cooling method that is reliable and effective in aggressively cooling a patient with EHS. When choosing a cooling modality for the treatment of EHS, the option that provides the maximum cooling rate that can be achieved with the available resources should be used (ie, CWI if possible, TACO method, and cold showering if temperature can reach the recommended $\left.0.15^{\circ} \mathrm{C} / \mathrm{min}\right) .{ }^{1}$ In conclusion, the findings from this critically appraised topic confirm CWI is the gold-standard treatment for EHS, but when this is not possible, the TACO method or another cooling method with an acceptable cooling rate of $0.15^{\circ} \mathrm{C} / \mathrm{min}$ or higher should be used on-site prior to transport to the emergency room. ${ }^{1}$ Death from EHS is preventable, and it is imperative for clinicians to have a plan for the prevention, recognition, and treatment of EHS for each individual setting.

\section{Acknowledgment}

The authors have no conflicts of interest to disclose.

\section{References}

1. Belval LN, Casa DJ, Adams WM, et al. Consensus statementprehospital care of exertional heat stroke. Prehosp Emerg Care. 2018;22(3):392-397. PubMed ID: 29336710 doi:10.1080/10903127. 2017.1392666

2. Casa DJ, DeMartini JK, Bergeron MF, et al. National Athletic Trainers' Association position statement: exertional heat illnesses. J Athl Train. 2015;50(9):986-1000. PubMed ID: 26381473 doi:10. 4085/1062-6050-50.9.07

3. Casa DJ, McDermott BP, Lee EC, Yeargin SW, Armstrong LE, Maresh CM. Cold water immersion: the gold standard for exertional heat stroke treatment. Exerc Sport Sci Rev. 2007;35(3):141-149. PubMed ID: 17620933

4. DeMartini JK, Ranalli GF, Casa DJ, et al. Comparison of body cooling methods on physiological and perceptual measures of hyperthermic athletes. J Strength Cond Res. 2011;25(8):20652074. PubMed ID: 21760549 doi:10.1519/JSC.0b013e3182259b1d

5. McDermott BP, Casa DJ, Ganio MS, et al. Acute whole-body cooling for exercise-induced hyperthermia: a systematic review. J Athl Train. 2009;44(2):84-93. doi:10.4085/1062-6050-44.1.84

6. Proulx CI, Ducharme MB, Kenny GP. Effect of water temperature on cooling efficiency during hyperthermia in humans. J Appl Physiol. 2003;94(4):1317-1323. PubMed ID: 12626467 doi:10.1152/ japplphysiol.00541.2002

7. Hosokawa Y, Adams WM, Belval LN, Vandermark LW, Casa DJ. Tarp-assisted cooling as a method of whole-body cooling in hyperthermic individuals. Ann Emerg Med. 2017;69(3):347-352. PubMed ID: 27865532 doi:10.1016/j.annemergmed.2016.08.428

8. Luhring KE, Butts CL, Smith CR, et al. Cooling effectiveness of a modified cold-water immersion method after exercise-induced hyperthermia. J Athl Train. 2016;51(11):946-951. PubMed ID: 27874299 doi:10.4085/1062-6050-51.12.07

9. Butts CL, Spisla DL, Adams JD, et al. Effectiveness of ice-sheet cooling following exertional hyperthermia. Mil Med. 2017;182(9): e1951-e1957. PubMed ID: 28885961 doi:10.7205/MILMED-D-1700057

10. Butts CL, McDermott BP, Buening BJ, et al. Physiologic and perceptual responses to cold-shower cooling after exercise-induced hyperthermia. J Athl Train. 2016;51(3):252-257. PubMed ID: 26942657 doi:10.4085/1062-6050-51.4.01

11. Adams WM, Hosokawa Y, Adams EL, Belval LN, Huggins RA, Casa DJ. Reduction in body temperature using hand cooling versus passive rest after exercise in the heat. J Sci Med Sport. 2016;19(11):936-940. PubMed ID: 27012727 doi:10.1016/j.jsams.2016.02.006

12. Casa DJ, Armstrong LE, Kenny GP, O’Connor FG, Huggins RA. Exertional heat stroke: new concepts regarding cause and care. Curr Sports Med Rep. 2012;11(3):115-123. PubMed ID: 22580488 doi:10.1249/JSR.0b013e31825615cc

13. Lopez RM, Casa DJ, McDermott BP, Stearns RL, Armstrong LE, Maresh CM. Exertional heat stroke in the athletic setting. Athlet Train Sports Health Care. 2011;3(4):189-200. doi:10.3928/1942586420101230-06

14. Rav-Acha M, Hadad E, Epstein Y, Heled Y, Moran DS. Fatal exertional heat stroke: a case series. Am J Med Sci. 2004;328(2): 84-87. PubMed ID: 15311166

15. Demartini JK, Casa DJ, Stearns R, et al. Effectiveness of cold water immersion in the treatment of exertional heat stroke at the Falmouth Road Race. Med Sci Sports Exercise. 2015;47(2):240-245. doi:10. 1249/MSS.0000000000000409

16. McDermott BP, Casa DJ, O'Connor FG, et al. Cold-water dousing with ice massage to treat exertional heat stroke: a case series. Aviat Space Environ Med. 2009;80(8):720-722. PubMed ID: 19653575 